Doug Geisler, Eva K. Grebel, and Dante Minniti, eds.

\title{
Infrared Imaging of the Arches Cluster - Adaptive Optics in the Densest Region of the Milky Way
}

\author{
Andrea Stolte, Eva K. Grebel \\ MPIA, Königstuhl 17, 69117 Heidelberg, Germany \\ Wolfgang Brandner \\ ESO, Karl-Schwarzschild-Str. 2, 85748 Garching, Germany
}

Donald F. Figer

STScI, 3700 San Martin Drive, Baltimore, MD 21218, USA

\begin{abstract}
The Arches cluster - located only 11' from the Galactic Center (GC) - is one of the densest and richest young star clusters in the Milky Way. With an age of only about $2 \mathrm{Myr}$, it is ideally suited to study massive cluster formation in an extreme environment. We find an IMF slope of $\Gamma=-0.77$ from 5 to $100 \mathrm{M}_{\odot}$, in good agreement with the results from HST/NICMOS from Figer et al. (1999). The limiting factor in the dense cluster center is crowding. With the new AO systems, high resolution analysis of the dense cluster region combined with very deep infrared photometry is available. We have analysed deep $\mathrm{H}$ and $\mathrm{K}^{\prime}$ images of the cluster center obtained with the GEMINI/Hokupa'a adaptive optics system. Colour-magnitude diagrams and the IMF are constructed from these data. A comparison with isochrones yields the mass function.
\end{abstract}

\section{Introduction}

During the past years, it has become clear that the slope of the initial mass function (IMF) in massive young star clusters (YCs) can deviate from the standard Salpeter value. A flat mass function with a slope of $\sim-0.7$ in comparison to the Salpeter value of $\Gamma=-1.35$ is found in massive Galactic clusters such as the Arches cluster (Figer et al. 1999, F99) and NGC 3603 (Eisenhauer et al. 1998). While Arches is evolving within an extraordinary environment, the Galactic Center, where effects such as tidal disruption, strong magnetic fields, high stellar densities, etc., may severely affect the evolution of a young star cluster, NGC 3603 is located in the Carina spiral arm, where a rather normal star forming environment is found.

Though those are two of the three most massive YCs found in the Milky Way, a flat IMF does not seem to be a general feature of massive YCs. The super-massive star cluster R136 in the Large Magellanic Cloud (LMC) displays a standard IMF with a slope of $\Gamma=-1.3$ (Hunter et al. 1996). To date, it is not yet clear which physical mechanisms determine the shape of the IMF. 


\section{Adaptive Optics Data}

High angular resolution observations are crucial to study the low-mass stellar content of dense star clusters. In addition to crowding, the high extinction in star-forming regions imposes strong constraints. For young clusters near the Galactic Center, the extinction may reach $A_{V} \sim 27$ (F99), but is much less severe in the near infrared $\left(A_{K}=3.1\right.$, assuming the extinction law from Rieke \& Lebofsky 1985). Due to the high spatial resolution of adaptive optics (AO) data from large telescopes such as Gemini, AO offers the possibility to determine the mass function far into the heart of a dense cluster, where the limiting factor is not the sensitivity, but crowding. We have analysed the Gemini science demonstration data of the Arches cluster to derive the mass function. $20^{\prime \prime} \times 20^{\prime \prime}$ images of the densest part of the cluster have been obtained under photometric conditions in $H$ and $K^{\prime}$. The effective resolution is $0^{\prime \prime} .2$ and 0.15 , with limiting magnitudes of 21 and 20 in $\mathrm{H}_{\text {and }} \mathrm{K}^{\prime}$, respectively.

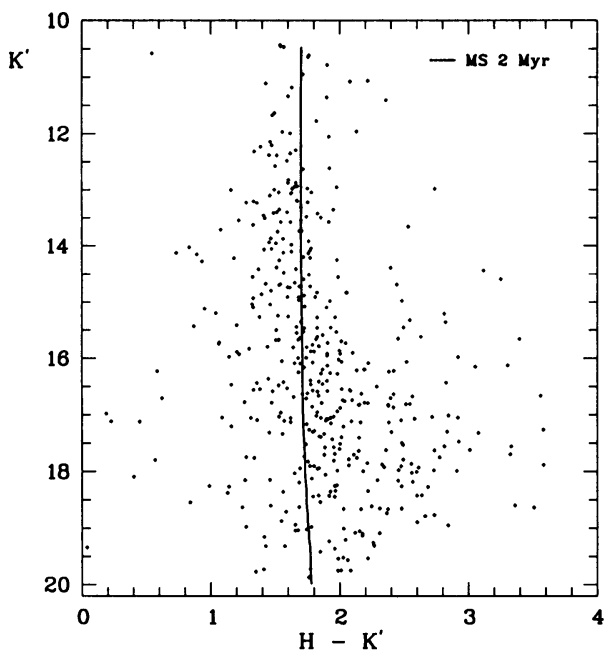

Figure 1. Colour-magnitude-diagram of the Arches center.

The 2 Myr main sequence isochrone (Lejeune \& Schaerer 2001) used for IMF derivation is plotted as a solid line.

\section{The Arches IMF}

We have derived the initial mass function (IMF) from the CMD using a 2 Myr isochrone from the Geneva set of models (Lejeune \& Schaerer 2001), displayed in Fig. 1, to transform luminosities into stellar masses. To derive the incompleteness fractions, artificial star experiments have been conducted on the deep $\mathrm{H}$ and $\mathrm{K}^{\prime}$ images. The loss from scatter of the main sequence due to the crowded field photometry has been accounted for. The IMF above $10 \mathrm{M}_{\odot}$ with a slope 
of $\Gamma=-0.77 \pm 0.15$ agrees very well with the IMF derived from HST data with $\Gamma=-0.7 \pm 0.1$ (F99). After correction for incompleteness, the steep part of the IMF continues down to $5 \mathrm{M}_{\odot}$, with no evidence for a turnoff or flattening.

Especially in comparison with massive LMC clusters such as R136, it is tempting to seek the answer to the deviating mass spectrum in Arches in the exceptional GC environment. High stellar densities, strong magnetic fields, collisions, etc., may cause the IMF to be skewed towards higher masses. On the other hand, tidal forces in the gravitational field near the GC may dynamically alter the spatial distribution of stars in GC YCs (Kim et al. 2000), yielding evolutionary changes hard to distinguish from intrinsic IMF properties. However, the effect of the environment on the IMF has still to be studied in detail.

The close agreement of our IMF slope to the HST result in Figer et al. (1999) shows that AO systems at 8-m class telescopes such as Gemini/Hokupa'a are capable of competing with space-born observations.

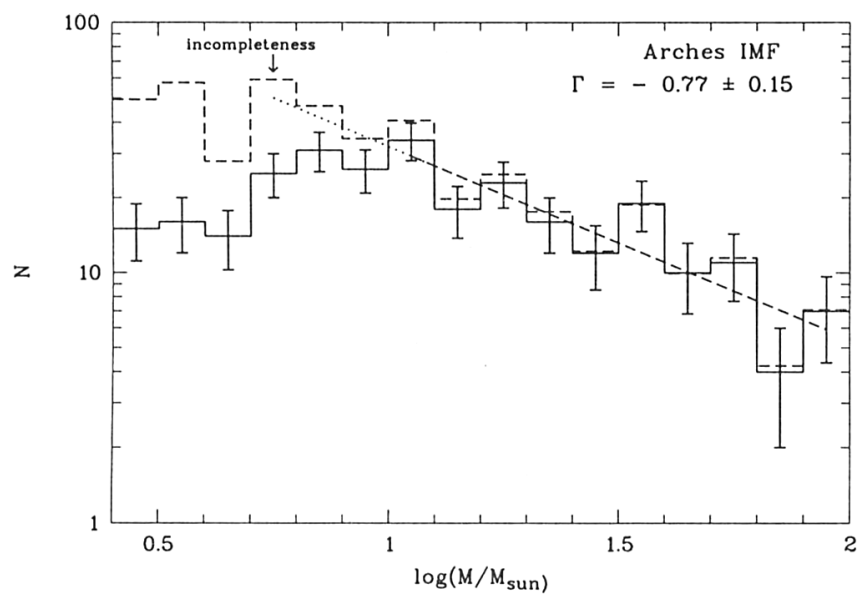

Figure 2. Arches IMF - dashed: completeness correction The line shows a linear regression for $\mathrm{M}>10 \mathrm{M}_{\odot}$.

\section{References}

Eisenhauer, F., Quirrenbach, A., Zinnecker, H., \& Genzel, R. 1998, ApJ, 498, 278

Figer, D. F., Kim, S. S., Morris, M., Serabyn, E., Rich, R. M., \& McLean, I. S. 1999, ApJ, 525, 750

Hunter, D. A., Shaya, E. J., Holtzman, J. A., Light, R. M., O’Neil, E. J., Jr., \& Lynds, R. 1995, ApJ, 448, 179

Kim, S. S., Figer, D. F., Lee, H. M., \& Morris, M. 2000, ApJ, 545, 310

Lejeune, T., \& Schaerer, D. 2001, A\&A, 366, 538

Rieke, G. H., \& Lebofsky, M. J. 1985, ApJ, 288, 618 